\title{
A Model of Cooperative Agent Based on Imitation and Maslow's Pyramid of needs
}

\author{
Le Guen Herve and Moga Sorin
}

\begin{abstract}
Recent works have addressed the problem of imitation in the framework of the interactions between two agents, whether humans or robots. We develop a model aiming at improving the self-organization of population of robots by relying on imitation. Imitations between the robots are regulated by a very simple model of emotional expression. The model is tested in the context of a simple task for the robots: to explore their environment to localize sources needed for their survival. Following a biology-inspired approach, imitation has been introduced within a population of autonomous agents, as bidirectional social needs, in line with the Maslow's Pyramid of needs [1]. In our model, imitation is integrated into a global architecture based on artificial neural networks. Running our simple and scalable model resulted in a significant increase of the population's survival rate and a decrease of the global amount of the average necessary movements of each agent.
\end{abstract}

\section{INTRODUCTION}

This paper presents our most recent results on the influence of imitation within a population of autonomous robots. More precisely, we attempt to find the minimum elements that can produce an efficient self-organization. Since the beginning of artificial intelligence, the role played by intrinsic individual intelligence has steadily decreased. Parallel research, in various fields, was aimed at proving the large amount of knowledge derived from others in individual intelligence. In psychology, the most famous experiments were conducted by Baldwin and Piaget cited by [2], [3], who showed the importance of imitation in infant development. In ethology, the collective intelligence of the ant, bee or primate is well known. A computational point of view emphasizes the obvious superiority of a distributed exploration of the set of possible states [4]. This can be a first step towards an efficient collective intelligence. The next question to solve is then the sharing of distributed knowledge. The fact that imitation is the possible root of communication and social learning [5], [6], [7] is well accepted now, as is shown by the amount of recent research in this area. One crucial reason for this growing interest by neuroscientists is probably the discovery of mirror neurons in primates [7]. The mirror neurons also fire when an action is performed, or when the same action, performed by a peer, is perceived. Indeed, the fact that the smallest functional component of the brain can behave in an imitative way constitutes a strong argument in favor of the role of imitation as the root of social abilities. Furthermore, mirror neurons are located in the equivalent of Broca's area in

Le Guen Herve and Moga Sorin are with the Institut TELECOM ; TELECOM Bretagne ;UMR CNRS 3192 Lab-STICC LUSSI, Technople Brest Iroise CS 8381829238 Brest Cedex 3 FRANCE (email: \{herve.leguen, sorin.moga\}@telecom-bretagne.eu).

This work was supported by the french ministry of research Grants the human brain, which contains the neurons most involved in language formulation. We therefore chose to consider in the present work that self-organization relies on imitation.

By studying the role of imitation in self-organization, we aim to supplement recent research on the various problems raised by imitation for robotics. Most of this research deals with the correspondence problem ([8], [9], [10], [11], [12], [13], [3] ): how can one get a humanoid robot to learn a sequence of actions by imitation and to succeed in linking the different body parts it observes with its own body components. In line with the analysis presented in [13], deal with the "how to imitate" problem. Other works [14], [15] were focused on how the intention can be perceived by the robot in order to guide the imitation. In other words, how the robot can infer the goal of the perceived action and how this goal can be used to select which part of an action is the most important.

Conversely, the present paper focuses on the "when to imitate" and "what to imitate" problems, thus implying a basic perception of intention. We assume that the correspondence problem (i.e the capacity of each robot to associate its own body components to body part it perceives) is solved. We propose a way to integrate a controlled imitation behavior within a population of autonomous robots. It is to a certain extent a holistic approach. Thus we solved two kinds of problems: first, how to integrate the imitation into the behavioral architecture of a robot or agent and, second, how to integrate it at the population level, i.e. how imitation needs to be adjusted to other basic behaviors (obstacle avoidance, random exploration, etc.), and how the target of imitation can be selected among a large number of possibilities. Finally, it should be noted that the context of the study is deliberately limited to a simple exploratory task: each robot has to localize a source corresponding to each of its vital needs in order to survive.

The first part of the paper presents the main phenomena concerning or relating to imitation. We have tried to determine a minimum set of low-level properties that leads to an efficient self-organization. This has been achieved in a large extent by studying an infant's abilities to imitate. These abilities include facial imitation [2], perception by the infant that he or she is being imitated [2], and empathy [16], [17], [18], [19], [20]. The interest in empathy sprung directly from the innate ability to imitate facial expressions and induced the study of emotions. Finally, the study of emotions was linked to the study of social needs as modeled by the Maslow's pyramid of motivations [1]. In a second part, we describe how each imitation phenomenon is modelled 
within an overall presentation of the robotic architecture and its implementation. The third part presents simulation results and compare them with the initial architecture.

\section{IMITATION}

Research of the minimal properties that an agent needs to possess in order to obtain efficient interactions leads naturally to consider what is innate or universal in human beings. Below are some observed phenomena related to imitation that present fall into these two categories.

\section{A. Innate imitation abilities}

According to Piaget, cited by [3], imitation is a sensorymotor learning process that leads to intelligence building. Earlier research focused on on delayed imitation, considered as a witness of representation appearance. But the studies undertaken by Meltzoff and Moore emphasized the innate ability to imitate. At least in the case of facial expression and simple gestures of tongue, mouth and fingers, the existence of natural mechanisms that facilitate imitation seems to be well-accepted now. Years of experiments led Meltzoff to the active intermodal mapping theory. This theory explains the phenomenon through the detection of similarities between the internal representations of, firstly, the visual perception of the demonstrator's face, and secondly, the internal representation of sensory motor perceptions. These representations, in the form of a map, could facilitate such detection. Although this mechanism is not yetfully understood, it constitute evidence of innate imitation abilities. In addition, it provides a clue to the strong implication of emotions in imitation phenomena. Moreover, the global linkage seems to be very complete and sharp: according to Meltzoff [2], the evolution of imitation is strongly linked to the development of all the personal aspects of self-knowledge. The more a baby or young infant learns about himself (motor abilities, goal-oriented actions, emotions, etc.), the more imitation enables him to make the association: "if it works this way for me, it must be the same for others". This process leads the infant to perceive very early that the actions of others may express intentions since he has intentions himself.

\section{B. The perception of the fact of being imitated}

An infant is very early able to perceive the fact that an adult or another infant is imitating him. Studies conducted by Meltzoff [2] produced some very informative results. Fourteen month-old babies were more interested by adults imitating them than by adults imitating video pictures of another baby. The two aspects of this perception of the fact of being imitated were separated into, firstly, synchrony between the baby's actions and the adult's actions and, secondly, the fact that these actions were identical. Experiments demonstrated that babies were more attracted by imitation than by merely synchronized actions. Moreover, they also tested the adults concerned in order to verify that they were really imitating.

\section{Empathy}

Although there was little interest in empathy a decade ago, its definition is hardly a subject of controversy. The common meaning given to the term is very similar to the basic definition used by scientists. Decety [16] suggests a basic definition: "an interaction between any two individuals, with one experiencing and sharing the feelings of the other." . Recent progress in neuroimaging permits a very precise description of the neural mechanisms involved in empathy. It is impossible here to describe all these mechanisms in full, but a superficial summary could be as follows: the activation of the same zones, except the primary sensory cortical area, involved in the same real experience with an intensity depending on each person's own regulation, and thanks to a top-down cognitive analysis of the other, and a bottom-up perception of the other person's emotional expression, both processes taking place at the same time. This last point is of particular interest to us, owing to its direct link with the innate spontaneous facial imitation phenomenon. In other words, the perception of facial expression influences the cognitive process. Most psychologists or neuroscientists agree that empathy generates a matching spontaneous facial expression. But studies of imitation, specially the ones by Meltzoff and Moore, demonstrate that this matching is already present in the newborn child. So the fact that the simple perception of facial expression plays a role in a very internal experience suggests that imitation cannot be studied without taking emotional expression in account. Section III will describe how this emotional interaction has been modelled. An explicit description of the chosen viewpoint regarding the emotions will be first given below.

\section{Emotions}

The role played by the expression of emotion in facial expressions has been outlined in the previous part through the empathy mechanisms and the imitation of facial expressions. Many studies have been carried out on the subject of human emotions which, owing to the great complexity of this phenomenon, and represent many different schools of thought (see [21] for a review). One point that does not provoke discussion is the almost universal nature of emotional expression. Studies [21] show a very low variability in the perception of facial expressions whatever the population concerned. In contrast to empathy, nobody has succeeded in giving a definition of emotions that achieves consensus even within a single disciplinary field. Nevertheless the results displayed by Rolls [22], which are based on recent technological advances in neuroimaging, are very informative and are in line with other parts of our paper. Rolls proposes that emotions are not only elicited by rewards and punishments arising from the current situation but are also influenced by situations that are expected. For instance, relief can be produced by the termination or non-appearance of a punishment. In the same logic, frustration, anger or sadness are the outcomes of the disappearance or mistaken expectation of a reward. Motivation is thus the expectation of reward and can be 
steered toward a secondary rewarding stimulus that becomes the goal for action. The model presented in this paper only focuses on the reward mechanisms and the emotions used are thus only related to motivation and reward, with the lack of satisfaction being considered as pain. So the findings on empathic response to pain have also been used for this case. Following the same reasoning as in the previous parts and linked to them, if emotions constitute an appraisal of the current situation and if those emotions are expressed in a universal manner, then the emotional expression will present characteristics well suited to serve as a criterion in target selection for imitation. Therefore, the fact that, firstly, new born children are able to perceive which facial expression is being made before them and, secondly, that young infants are very early able to make the hypothesis that their own internal state associated with such acts could be identical to that of another person doing the same thing, leads us to consider that these mechanisms are involved in communication modulation.

\section{E. Maslow's pyramid of needs}

We have presented above the phenomena involved in imitation that we have chosen to model. The problem is to integrate all these processes into a global architecture. As there exist no neurobiological data, we turned towards psychology and a well established model, namely Maslow's pyramid of needs[1]. This model has been used successfully many times and, despite the date of its publication, it is still used in numerous domains. According to this model, a human being's motivations follow a hierarchy (Fig.1). Although the influence of each particular need varies from one person to another the principle is the same. There are two main classes of needs. The most important needs are called primary needs. Put briefly, they are made up of the physiological needs such as eating, breathing, maintaining the bodily temperature, escaping predators, and so on. According to the theory, if a deeper need is not satisfied, needs higher up in the hierarchy cannot be a source of motivation for an individual [1].

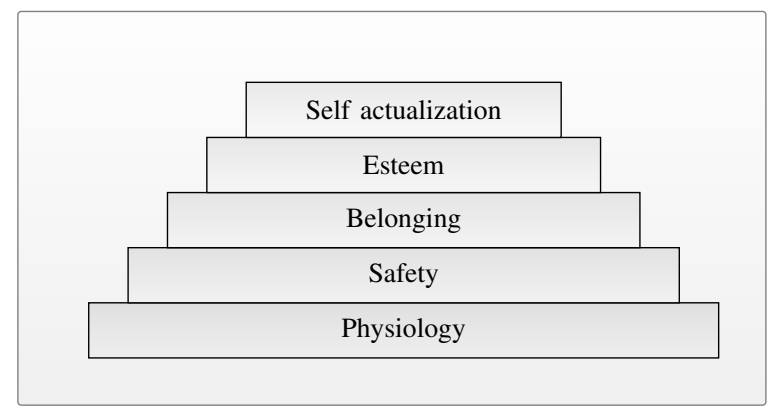

Fig. 1. Maslow's Pyramid of needs

The second class of needs are called secondary needs. They constitute the social aspect of human motivation. First there is the gregarious instinct of human beings: the need to belong. Human beings need friendship, love, social relationships. This need is the need to belong to a social group, an association, or any kind of social network. Next is the need for esteem. This need is in fact bidirectional: human beings need first of all to feel esteem for others, but also to receive esteem from others. These two needs have been interpreted, in our context, as the need to imitate, and the need to be imitated. They are based, firstly, on the decision process of the agents(explained in next section) and, secondly, on the two different kinds of satisfaction, presented with their properties in the previous section. The fact that rewards may be shared thanks to an empathic response implies that a reward must be expected after some social interchange. Consequently, as the fact of being imitated is perceived by the agent concerned and as imitation is likely to induce empathic satisfaction, it seems logical to model them as needs, that is to say, as expected rewards. Finally, it can be added that implementing imitation as a social need is facilitated by the architecture of the initial model [23], which is based on a subsumption architecture [24]. This is also a hierarchical architecture composed of basic behaviors. The implementation of these needs will be described in greater detail in the next section.

\section{MODEL}

The model was based on an existing model of an autonomous robot with goal-oriented navigation and imitation capabilities [25],[26],[27]. Its goals are derived from internal variables that have to be maintained in a comfort area. The values of these variables decrease in time. The robot population's task is to explore an unknown environment and to localize sources corresponding to its different intrinsic needs. Its survival depends upon the satisfaction of these needs by discovering the different locations of the sources. In order to link them with Maslow's pyramid, these individual needs are called primary needs. In reality, we refer to a need when the corresponding internal variable falls below a certain threshold. More generally, this model is structured as a Brooks architecture [24] with a hierarchy of different behaviors. These behaviors are, in the first place, obstacle avoidance, secondly goal-oriented navigation, thirdly imitation, and lastly random exploration. And so, at each computation step, if a behavior is either unnecessary or impossible, then the next one is attempted (Fig. 3). To these four basic behaviors correspond four modules. The obstacle avoidance module relies on a Braitenberg-like mechanism. The goaloriented navigation module is based on a biologically inspired model that makes use of the studies by O'Keefe and Nadel [28] on the hippocampus and cognitive maps. Thanks to this model, a representation of the environment in the form of a graph is memorized according to the novelty aspect of a location by comparing visual landmarks positions[25], [29]. Such a graph allows goal-directed navigation by propagating motivation from a source corresponding to a real need and to the current location of the robot. The graph is in fact implemented as an artificial neural network. This network is composed of two different layers, one that can be seen as a sensory cortex is used for self-localization and the other to propagate the motivation. 


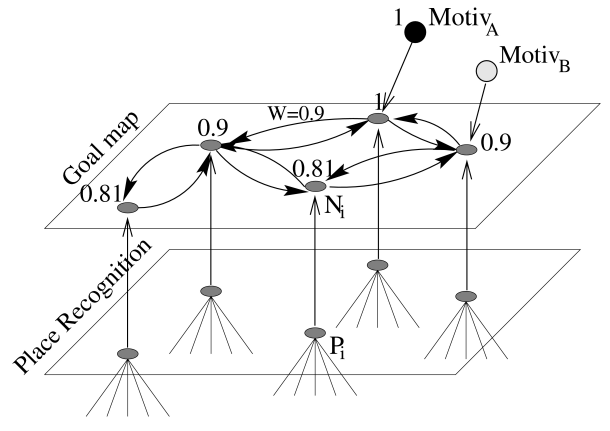

Fig. 2. The goal-oriented navigation model [29]

The neurone where global activation, that derives from both localization and motivation, is maximum, becomes the goal for next action. Robots can thus retrieve the source location by following the motivation gradient (Fig.2). Obviously, this kind of behavior is only possible when a source location is known. If that is not the case, the robot switches to the next behavior module. The next module is the imitation module, implemented in this initial model as a simple following imitation behavior, i.e. the robot is able to follow a peer's displacement. This behavior was possible when other robots were present in the field of perception. Lastly, the exploratory module carries out a random exploration of the environment.

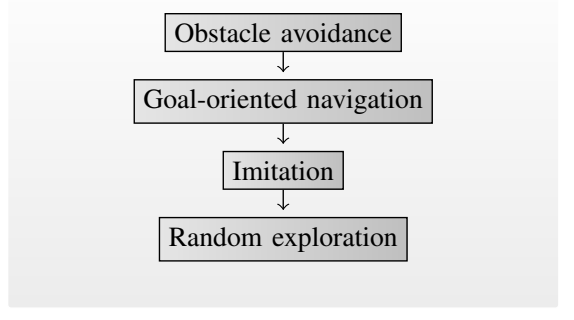

Fig. 3. The behavioral architecture

For more explanation about this model, see [23], [29]. In this paper we present modifications concerning the imitation module only. As stated earlier, the imitation behavior was divided into three new natural needs called secondary needs. To be more precise, they are the acceptance need, the esteem for others need and the esteem by others need. These needs induce three new internal variables that, if they are not satisfied, decrease in time by the following dynamic:

$$
V^{i}{ }_{t+1}=V^{i}{ }_{t} \cdot(1-\alpha)
$$

Where $V^{i}$ is the $i^{t h}$ secondary variable, and $0<\alpha<1$.

To each secondary variable corresponds a specific basic behavior hierarchically integrated into the overall architecture. As a result, they function at the same level as the others, such as obstacle avoidance or random exploration.

In addition, an external signature has been added in order to model emotional expression. This signal can be perceived by the others agents so as to modulate imitation events within the whole population by giving a new target selection criterion for imitation.

\section{A. The emotional signature}

As already explained, the emotional signature expresses the current state and the expected state of the agent. Therefore, the motivational state that constitutes the expected satisfaction is also expressed in this signature. An agent in its comfort area displays a neutral signature whereas an internal variable below a certain threshold induces pain. That pain will cause a potential empathic response that is likely to incite another agent to move toward a known source. On the other hand, a motivated agent is likely to provoke an attractive empathic response. Indeed, the fact that making a decision about the action to perform depends upon the motivation, renders attractive the perception of the motivation with empathy. Consequently, keeping a motivated agent in its own field of perception becomes a source of motivation. This kind of reasoning leads to the selection of the imitation target according to its motivated state. We posit here that a motivated agent is thus more attractive than a satisfied one and that an marked lack of satisfaction induces pain. This signature expresses the self-appraisal of an agent, according to the following rule:

$$
S=\left\{\begin{array}{l}
50 \text { if } \forall i, V_{i}>s \\
50 *\left(1+M^{\alpha}\right)-\left(V_{4}+V_{5}\right) / 4 \text { si } M>K B \\
\min _{i}\left\{V_{i}\right\} \text { else }
\end{array}\right.
$$

Where $S$ is the agent signature, $0<M<1$ its motivation $V^{i}$ is the $i^{t h}$ internal variable, $K B$ a noise constant, and $\alpha$ serves only to enhance the contrast. To be more precise, when several source locations propagate motivation, the one with the lowest internal variable provokes the highest motivation. Consequently, it is the only one that influences the selection of the action.

\section{B. The acceptance need}

This natural inclination has been interpreted as a gregarious instinct. It can also be perceived as the outcome of a social learning process produced by the combination of past satisfaction obtained with the help of a peer or by the empathic propagation of another satisfaction. Indeed, the empathic response to the perception of obtaining a reward also induces satisfaction in the observer. In fact, we make here the hypothesis that an innate empathic capability can serve as a kind of catalyst of social attraction. Empathy induces an association between satisfaction and the presence of others. This presence is considered so necessary to social satisfaction that it could be modelled as a need in itself. The association between a pleasure and a location is a well known result. The conditioned place preference technique is commonly used in ethology to illustrate this mechanism. Therefore, the sources that increase the associated internal variable are locations where a high density of population 
is perceived. An agent can thus aim for these meeting places by using its goal-directed navigation capability. In other words, the learned meeting location also propagates motivation through the motivational neural layer in order to guide the action selection. Moreover, the variable is set at its highest level each time the number of agents perceived reaches a percentage (70\%) of the expected number, as initially known when the meeting place was first learned.

\section{The esteem for others need}

Here our hypothesis is that an imitation event is only possible if the imitator's signature is lower than the demonstrator's one. When several agents have a higher signature, the one with the highest is chosen as demonstrator. This decision mechanism is consistent with the fact that the global action selection criterion is the level of motivation. Following the agent with the strongest motivation will produce the highest motivated state, induced by the empathic response of the imitator. In this case, using this criterion to make a decision is equivalent to considering the influence of empathy. The corresponding internal variable is increased when an imitation leads to satisfaction, i.e. when an agent reaches a source by following another during a fixed number of time step.

Lastly, the fact that the motivational state is perceived, can be related to the other studies mentioned in the introduction, where the motivation is a distance to the goal. This kind of perception does not constitute a perception of intention but the perception that an agent intends to do something and of how distant from its goal it is. Perceiving how far the demonstrator is from the goal will help to decrease the number of possible goals of an observed action.

\section{The need for esteem by others}

This need has been interpreted as the need to be imitated. As with the previous need, action selection follows motivation intensity through empathy. The pain or the need, felt by an observed agent, activates the observer's motivational neural layer with the result that it moves toward one of its known sources. That is to say, the lower the emotional signature, the more it impacts on the internal state of the others. This impact will generate the observer agent's motivation; therefore, the more an agent is in difficulty, the more it is attractive. In short, internal primary variables decrease by the difference between the neutral value and the value of the signature of the agent feeling pain. Consequently, the decrease in primary variables induces motivation for the observer. This primary motivation has priority in the hierarchy of needs, but only in the presence of the agent in pain. Should this agent disappear, the motivation falls and the move toward the source stops. The reduction of variables is modulated by a parameter that can be regarded as the self-regulation process of empathy described by Decety in [16]. An agent considers himself as imitated when another agent is behind him, that is to say, perceived with an azimuth higher than $\pi / 2$, during a fixed number of consecutive time step. In order to reduce the average number of displacements, a new basic behaviour implanted in the robots is to wait. It is linked to the esteem by others need and is used when an agent is not attractive enough to be imitated by the agent in the field of perception. As for the dynamic aspect of the resource needed, an agent does not increase the probability of its meeting another agent by moving. Owing to the acceptance need, it is likely to be located at a meeting place. The likelihood of meeting is improved by his waiting there. This is a passive response to the need for esteem. Moreover, this passive response maintains the synchrony between the imitator and the demonstrator. If the imitator gets too far from the demonstrator it has to wait since its motivation falls immediately.

Lastly, the satisfaction of the esteem for others need occurs when a source is reached. But in this case, this satisfaction comes from the empathic response to the agent's reward. The demonstrator associates this empathic satisfaction with its perception of the fact that it has been imitated. In other words, this mechanism implies the perception of being imitated. This phenomenon must also be incorporated into the set of minimal properties for the implementation of such a model.

\section{E. Conclusion}

Expressing such behaviors in the form of needs presents many advantages. Although at this stage the implementation is very simple, it offers the possibility of evolving towards social learning by extending the current neural layers. Such an implementation could make the association between signals that are the most relevant for efficient social interactions. But here our main objective is to propose a global synthesis of recent neuroscientific research in the form of a minimal set of biology-inspired properties. Moreover, the aim of this paper is also to facilitate the global integration of these properties. The next part will show that it enhances the efficiency of exploration by a population of autonomous robots. Lastly it should be emphasised that all the changes presented here have little influence on the overall computational complexity. Indeed, to a large extent they make use of the existing navigation mechanisms.

\section{RESULTS AND COMPARISON WITH THE INITIAL MODEL}

\section{A. Simulations}

The results presented here have been obtained by means of computer-generated simulations. The simulator used (Fig.4) relies on a client server architecture. The server constitutes the environment and agents are clients. The server has been implemented in java whereas agents are $\mathrm{C}$ programs. They communicate thanks to tcp connections that allows simulations to run on a parallel architecture, in other words, the server and the agents can run on different computers. The most recent version of the simulator could enable simulations with at least thousands of agents in a reasonable time.

To give a more precise description, this a two dimensional environment divided into squares. A map can be endowed 
with sources, obstacles and landmarks. The size of the perception field of every agent is a parameter. They can thus perceive another agent or a source if they are located in their neighborhood contrary to landmarks. Landmarks are perceived from a large distance except if there is an obstacle between them and the agent. That the reason why the localization system relies on them. In a real robotic context, the visual process must be able to detect these landmarks on its own, but here we assume this is already performed.

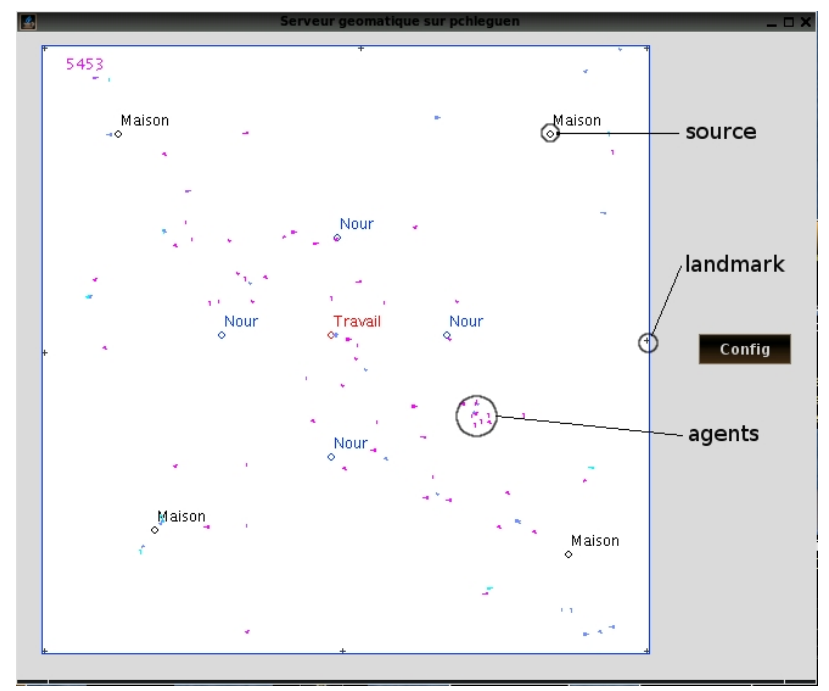

Fig. 4. The simulator

The architecture use a synchronous communication protocol. At each simulation time step, the java server send to every agent a message that contains the set of perceptions corresponding to its position. To enhance the simulation speed, these perceptions are calculated in advance for each square. They are composed of a set of perceived landmarks, obstacles and source associated each with the angle made with a virtual north used as a reference. These data are completed with the set of the others agents located in the perception scope, associated with their emotional signature and their angle with the north. These sets of localized informations are the only data used by the agents. As described in previous section, the azimuth of the landmarks are sufficient to generate a cognitive map of the environment in the shape of a graph. The global decision process produced an elected action that is sent back to the server. As this is a synchronous protocol, the server wait all the agents have sent their action message before sending next perceptions messages. Once the server received all the actions, it displays the new positions, computes the agents perceived from each position and then send the next perceptions.

To complete the description, it can be added that, first, dynamic sources are also managed by the server. A source can thus have a limited capacity and disappear once it is empty. The server then generate a new one at a randomly chosen location. This kind of simulations emphasizes how an organization already shaped can accomplish the exploration task, and how it adapts to the new locations. But, the results presented here are not concerned with this property. Second, it is also possible to view an agent cognitive map and its activity through a GUI.

The outcomes of a simulations are log files that allow to complete a visual appraisal of the global behavior of the population (individual motivational states are also displayed through agents color). These files contain the internal states of every agent at each simulation time step. The results presented here are based on the analyze of them.

The analysis of the simulations seeks to illustrate the reliability and adaptability of the model. Therefore, it only shows the survival rate and the amount of movement in different types of situation. These results are then compared with two other kinds of agent: reactive and initial. Reactive agents are endowed with navigation abilities but with no imitation behavior. Initial agents constitute the starting point of our study. Their imitation behavior is not a need but rather a basic behavior of integrated into a subsumption architecture. This basic behavior in particular enhanced the survival rate of a population of agents with great adaptability and scalability[23]. Agents following the model presented in this paper are called Maslow agents. In addition, the field of perception was given a very low value. Results presented here only aimed to validate the Maslow model, but don't constitute a detailed analysis of the resulting selforganization. However, they prove a real improvement in cooperation.

\section{B. Survival rate}

Maslow agents were tested with a different density of sources and a different density of populations.

\begin{tabular}{lllll}
\hline Type & \multicolumn{4}{c}{ Size of population } \\
of agents & 5 & 10 & 30 & 100 \\
\hline Reactives & $58.0 \%$ & $58.0 \%$ & $58.0 \%$ & $58.0 \%$ \\
Initials & $66.0 \%$ & $75.5 \%$ & $90.9 \%$ & $98.7 \%$ \\
Maslow & $\mathbf{9 1 . 5 \%}$ & $\mathbf{9 8 . 0 \%}$ & $\mathbf{1 0 0 \%}$ & $\mathbf{1 0 0 \%}$ \\
\hline
\end{tabular}

TABLE I

SURVIVAL RATE IN AN ENVIRONMENT ENDOWED WITH 3 SOURCES

\begin{tabular}{lllll}
\hline $\begin{array}{l}\text { Type } \\
\text { of agents }\end{array}$ & 5 & 10 & 30 & 100 \\
\hline Reactives & $76 \%$ & $76 \%$ & $76 \%$ & $76 \%$ \\
Initials & $100 \%$ & $100 \%$ & $100 \%$ & $100 \%$ \\
Maslow & $\mathbf{1 0 0 \%}$ & $\mathbf{1 0 0 \%}$ & $\mathbf{1 0 0 \%}$ & $\mathbf{1 0 0 \%}$ \\
\hline
\end{tabular}

TABLE II

SURVIVAL RATE IN AN ENVIRONMENT ENDOWED WITH 6 SOURCES

These experiments (table I and table II) showed a real enhancement of the global survival rate even with a very small population of five agents wherein communications are not frequent. Conversely, a high density of agents isn't a problem despite the number of potential meeting locations become high. In fact, the number of meeting location and 
its dynamic will the point that we'll focuse on. It constitutes a crucial point concerning the global resulting organization shape. Simple modifications seem to produce an interesting variability in outcoming organization. At present, the model described here strives for a centralized organization, but a more precise study is in progress. Obviously reactive agents are not impacted by the population size since there is no interactions between them.

\section{Quantity of movements}

\begin{tabular}{lllll}
\hline $\begin{array}{l}\text { Type } \\
\text { of agents }\end{array}$ & 5 & 10 & 30 & 100 \\
\hline Reactives & 365 & 365 & 80 & 399 \\
Initials & 363 & 369 & 380 & 400 \\
Maslow & $\mathbf{3 1 6}$ & $\mathbf{2 9 3}$ & $\mathbf{2 7 3}$ & $\mathbf{2 6 1}$ \\
\hline
\end{tabular}

TABLE III

QUANTITY OF MOVEMENTS IN AN ENVIRONMENT WITH 3 SOURCES(PIXELS PER TIME STEP)

\begin{tabular}{lllll}
\hline $\begin{array}{l}\text { Type } \\
\text { of agents }\end{array}$ & 5 & \multicolumn{4}{c}{ Size of population } \\
\hline Ractives & 355 & 365 & 36 & 100 \\
\hline Initials & 357 & 358 & 367 & 399 \\
Maslow & $\mathbf{3 1 7}$ & $\mathbf{2 8 9}$ & $\mathbf{2 5 4}$ & $\mathbf{2 4 5}$ \\
\hline
\end{tabular}

TABLE IV

QUANTITY OF MOVEMENTS IN AN ENVIRONMENT WITH 6 SOURCES(PIXELS PER TIME STEP)

A significant gain in average movement (table III and table IV) of between $20 \%$ and $40 \%$ depending on the situation was observed. In addition, the model showed good adaptation abilities since this gain increased with the population density. This is consistent with the fact that a smaller proportion of the population is needed to explore the environment. More precisely, the observed phennomenon is an increase of the number of waiting situations since the primary needs are quickly satisfied. Waitings produce clustering of agents and are the mechanism that contribute to keep a meeting point alive. Thus agents arriving in one of these locations have, first, a higher probability to learn this place as a meeting point, and second, are quickly teached by the waiting agents. The waiting basic behavior strongly impacts the organizational dynamic by reinforcing the locations where interactions have a high likelihood to happend. Moreover this behavior reduces the use of power in a robotic context.

\section{Diffusion of information in terms of density of population}

Lastly, the collective level of knowledge was taken into account. To this end, the percentage of the population that knew all the sources in the environment was measured and its evolution compared for different population densities.

Knowledge propagation increases with the density of population. Despite the impact of density, communication

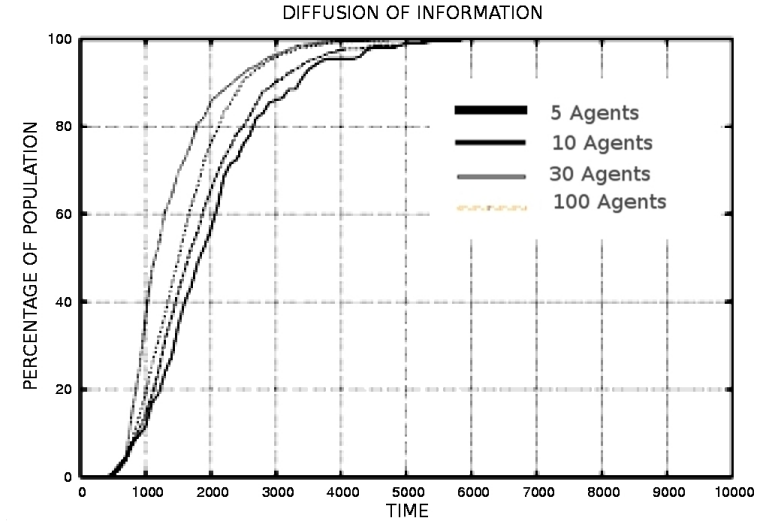

Fig. 5. Diffusion of information function of density of population

frequency plays the main role in the control of this dynamic. This frequency depends greatly on the social needs that are settable. Future study may lead to the optimisation of different social parameters depending on the situation, knowing that high communication frequency reduces exploration but increase the information diffusion.

\section{CONCLUSIONS AND FUTURE WORKS}

Based on a synthesis of different recent research projects dealing with the study of emotions and imitation, we have proposed a holistic approach to the implementation of imitation in autonomous agents. The tests we conducted show that our modelling approach, which uses recent advances in neuroscience, has successfully increased the robot cooperation efficiency. Our architecture permits a simple and scalable model of agent. These developments should prove usefull in applications such as swarm piloting, data mining or ad-hoc networks. Some organizational analysis is presently being carried out to determine the optimal parameters for a given situation and the different shapes of resulting organizations. A real robotic implementation is currently also in progress in order to validate the model's reliability in real constraints.

\section{REFERENCES}

[1] A. H. Maslow. A theory of human motivation. Psychological Review, (50):370-396, 1943.

[2] J. Nadel and J. Decety. Imiter pour dcouvrir l'humain (in french). Presses Universitaires de France, 2002.

[3] S. Moga. Apprendre par imitation: une nouvelle voie d'apprentissage pour les robots autonomes (in french). $\mathrm{PhD}$ thesis, Cergy-Pontoise university and Universitatea Politehnica TIMISOARA, 2000.

[4] Jacques Ferber. Les Systèmes multi-agents: Vers une intelligence collective (in french). Dunod, January 2007.

[5] V. Gallese, C. Keysers, and G. Rizzolatti. A unifying view of the basis of social cognition. Trends Cogn Sci, 8(9):396-403, September 2004.

[6] M. A. Arbib. The Mirror System, Imitation, and the Evolution of Language. The MIT Press, 2002.

[7] G. Rizzolatti and M. A. Arbib. Language within our grasp. Trends in Neurosciences, 21(5):188-194, 1998.

[8] A. Alissandrakis, C.L. Nehaniv, and K. Dautenhahn. Imitation with alice: learning to imitate corresponding actions across dissimilar embodiments. Systems, Man and Cybernetics, Part A, IEEE Transactions on, 32(4):482-496, Jul 2002. 
[9] A. Alissandrakis, C.L. Nehaniv, and K. Dautenhahn. Correspondence mapping induced state and action metrics for robotic imitation. Systems, Man, and Cybernetics, Part B, IEEE Transactions on, 37(2):299307, April 2007.

[10] Rajesh P. N. Rao Aaron P. Shon, Joshua J. Storz. Towards a real-time bayesian imitation system for a humanoid robot. IEEE International Conference on robotics and automation, 2007.

[11] A. Alissandrakis, C.L. Nehaniv, and K. Dautenhahn. Synchrony and perception in robotic imitation across embodiments. Computational Intelligence in Robotics and Automation, 2003. Proceedings. 2003 IEEE International Symposium on, 2:923-930 vol.2, 16-20 July 2003.

[12] M. Elshaw, C. Weber, A. Zochios, and S. Wermter. An associator network approach to robot learning by imitation through vision, motor control and language. Neural Networks, 2004. Proceedings. 2004 IEEE International Joint Conference on, 1:-596, 25-29 July 2004.

[13] K. et al. Dautenhahn. Imitation in animals and artifacts. MIT Press, Cambridge MA, 2002.

[14] B. Jansen and T. Belpaeme. A model for inferring the intention in imitation tasks. The 15th IEEE International Symposium on Robot and Human Interactive Communication, 2006.

[15] B. Jansen and T. Belpaeme. A model for inferring the intention in imitation tasks. Robot and Human Interactive Communication, 2006. ROMAN 2006. The 15th IEEE International Symposium on, pages 238-243, Sept. 2006.

[16] Jean Decety and Philip L. Jackson. A social-neuroscience perspective on empathy. Current Directions in Psychological Science, 15:5458(5), April 2006.

[17] Michael A. Arbib and Jean-Marc Fellous. Emotions: from brain to robot. Trends in Cognitive Sciences, 8(12):554-561, December 2004.

[18] J. Decety and J. Grèzes. The power of simulation: Imagining one's own and other's behavior. Brain Res, 1079(1):4-14, March 2006.

[19] Tania Singer. The neuronal basis and ontogeny of empathy and mind reading: Review of literature and implications for future research. Neuroscience \& Biobehavioral Reviews, 30(6):855-863, 2006.

[20] M. Jabbi, M. Swart, and C. Keysers. Empathy for positive and negative emotions in the gustatory cortex. NeuroImage, 34(4):17441753, February 2007.

[21] C. Belzung. Biologie des emotions (in french). De Boeck University, 2007.

[22] E. T. Rolls. Memory systems in the brain. Annual Review of Psychology, 51:599-630, 200.

[23] Ph. Laroque, N. Cuperlier, and P. Gaussier. Impact of imitation on the dynamics of animat populations in a spatial cognition task. In Proc. of IAS-8, Intelligent Autonomous Systems, 2004.

[24] R. Brooks. A robust layered control system for a mobile robot. Robotics and Automation, IEE Journal of, 2:14-23, 1986.

[25] J.P. Banquet, P. Gaussier, A. Revel, and Y. Burnod. A hippocampal model of visually guided navigation as implemented by a mobile agent. Neural Networks, 2000. IJCNN 2000, Proceedings of the IEEE-INNSENNS International Joint Conference on, 2:41-46 vol.2, 2000.

[26] J.P. Banquet, P. Gaussier, M. Quoy, A. Revel, and Y. Burnod. Place cells, maps and navigation strategies: processing steps of the corticohippocampal system. Neural Networks, 2002. IJCNN '02. Proceedings of the 2002 International Joint Conference on, 2:1433-1438, 2002.

[27] J.P. Banquet, P. Gaussier, A. Revel, S. Moga, and Y. Burnod. Sequence learning and timing in hippocampus, prefrontal cortex, and accumbens. Neural Networks, 2001. Proceedings. IJCNN '01. International Joint Conference on, 2:1053-1058 vol.2, 2001.

[28] J. O'Keefe and L. Nadel. The Hippocampus as a cognitive map. Oxford University Press, 1978.

[29] M. Quoy, S. Moga, and P. Gaussier. Dynamical neural networks for planning and low-level robot control. Systems, Man and Cybernetics, Part A, IEEE Transactions on, 33(4):523-532, July 2003.

[30] P. Andry, P. Gaussier, S. Moga, J.-P. Banquet, and J. Nadel. Learning and communication via imitation: An autonomous robot perspective. IEEE Transactions On Systems, Man, And Cybernetics, 31, 2001.

[31] P. Andry, P. Gaussier, S. Moga, A. Revel, and J. Nadel. Imitation: learning and communication. In 6th conference of Society for Adaptive Behavior, SAB2000, 2000.

[32] Ignazio Infantino Antonio Chella, Haris Dindo. Learning high-level manipulative tasks through imitation. The 15th IEEE International Symposium on Robot and Human Interactive Communication, 2006.

[33] Michael A. Arbib, James Bonaiuto, and Edina Rosta. The mirror system hypothesis: From a macaque-like mirror system to imitation, pages 3-10. 2006.
[34] T. Belpaeme, B. De Boer, B. De Vylder, and B. Jansen. The role of population dynamics in imitation. Pas publi, apparemment, 2004.

[35] A. Billard, Y. Epars, Gordon Cheng, and S. Schaal. Discovering imitation strategies through categorization of multi-dimensional data. Intelligent Robots and Systems, 2003. (IROS 2003). Proceedings. 2003 IEEE/RSJ International Conference on, 3:2398-2403 vol.3, 27-31 Oct. 2003.

[36] Aude Billard and Gillian Hayes. Drama, a connectionist architecture for control and learning in autonomous robots. Adaptive Behavior, 7:35-64, 1999.

[37] A. Billard, A.J. Ijspeert, and A. Martinoli. A multi-robot system for adaptive exploration of a fast changing environment: probabilistic modelling and experimental study. Connection Science, 11(3-4):359379, 1999.

[38] Richard W. Byrne. Detecting, Understanding, and Explaining Animal Imitation Chapter 9 in Perspectives on imitation: from mirror neurons to memes, volume 1. MIT Press, Cambridge MA, 2005.

[39] C. Caldwell and A. Whiten. Evolutionary perspectives on imitation: is a comparative psychology of social learning possible? Animal Cognition, 2002.

[40] R. Fukano, Y. Kuniyoshi, and A. Nagakubo. A cognitive architecture for flexible imitative interaction using tools and objects. Humanoid Robots, 2006 6th IEEE-RAS International Conference on, pages 376381, 4-6 Dec. 2006

[41] Vittorio Gallese. Intentional attunement: A neurophysiological perspective on social cognition and its disruption in autism. Brain Research, 1079(1):15-24, March 2006.

[42] Vittorio Gallese and David Freedberg. Mirror and canonical neurons are crucial elements in esthetic response. Trends in Cognitive Sciences, 11(10):411+, October 2007.

[43] Vittorio Gallese and Alvin Goldman. Mirror neurons and the simulation theory of mind-reading. Trends in Cognitive Sciences, 2(12):493501, December 1998.

[44] I. Goga and A. Billard. Attention mechanisms for the imitation of goal-directed action in developmental robots. In EPIROB 2006, 2006.

[45] Auke Jan Ijspeert, Alcherio Martinoli, Aude Billard, and Luca Maria Gambardella. Collaboration through the exploitation of local interactions in autonomous collective robotics: The stick pulling experiment. Auton. Robots, 11(2):149-171, 2001.

[46] C. M. Johnson. Distributed primate cognition: a review. Animal Cognition, V3(4):167-183, March 2001.

[47] C. Keysers, B. Wicker, V. Gazzola, J. L. Anton, L. Fogassi, and V. Gallese. A touching sight: Sii/pv activation during the observation and experience of touch. Neuron, 42(2):335-346, April 2004.

[48] Y. Kuniyoshi, Y. Yorozu, M. Inaba, and H. Inoue. From visuo-motor self learning to early imitation-a neural architecture for humanoid learning. Robotics and Automation, 2003. Proceedings. ICRA '03. IEEE International Conference on, 3:3132-3139 vol.3, 14-19 Sept. 2003.

[49] M. Lopes, F.S. Melo, and L. Montesano. Affordance-based imitation learning in robots. Intelligent Robots and Systems, 2007. IROS 2007. IEEE/RSJ International Conference on, pages 1015-1021, Oct. 29 2007-Nov. 22007.

[50] G. Rizzolatti, L. Fogassi, and V. Gallese. Motor and cognitive functions of the ventral premotor cortex. Curr Opin Neurobiol, 12(2):149-154, April 2002.

[51] Magali J. Rochat, Elisabetta Serra, Luciano Fadiga, and Vittorio Gallese. The evolution of social cognition: Goal familiarity shapes monkeys' action understanding. Current Biology, 18(3):227 - 232, 2008. 